\title{
Tagung: Lernen durch entwicklungspolitische Freiwilligendienste ${ }^{1}$
}

\author{
Julia Keller \\ Projektkoordinatorin für Globales Lernen beim Dachverband Entwicklungspolitik \\ Baden-Württemberg (DEAB), Stuttgart | julia.keller@deab.de
}

Ein Freiwilligendienst im globalen Süden - und dann? Entwicklungspolitische Freiwilligendienste bieten eine Chance für Lernprozesse, bei denen junge Menschen durch persönliche Erfahrungen, Erlebnisse und Eindrücke im Sinne des Globalen Lernens Kompetenzen erwerben, sich hin zu global verantwortlichen WeltbürgerInnen zu entwickeln. Wie können diese Lernprozesse von der Vorbereitung von Freiwilligendiensten bis hin zur RückkehrerInnenarbeit durch pädagogische Begleitung begünstigt werden? Wie können RückkehrerInnen diese Lernerfahrungen nach ihrer Rückkehr in Deutschland einbringen? Welche Vernetzungsmöglichkeiten haben Entsendeorganisationen und Nichtregierungsorganisationen (NROs) der entwicklungspolitischen Inlandsarbeit, um langfristiges Engagement von RückkehrerInnen zu fördern? Mit diesen und anderen Fragen setzten sich die rund 30 RückkehrerInnen, VertreterInnen von Entsendeorganisationen und entwicklungspolitischen NROs bei der Tagung „Lernen durch entwicklungspolitische Freiwilligendienste“ auseinander. Die Veranstaltung fand am 22. November 2013 in Stuttgart statt und wurde vom Dachverband Entwicklungspolitik Baden-Württemberg $(D E A B)^{2}$ zusammen mit dem forum für internationale entwicklung+planung (finep) ${ }^{3}$ veranstaltet. Im Rahmen des europäischen Projektes Zurück für die Zukunft wurde die Tagung finanziell gefördert durch die Europäische Union.

In einem ersten Teil wurden drei Ansätze vorgestellt, wie Lernprozesse und Engagement von (ehemaligen) Freiwilligen befördert werden können. Das Eine Welt Netz NRW ist seit 2008 selbst weltwärts-Entsendeorganisation. Hier zeigte Miriam Elsinghorst auf, wie Lernprozesse bei Freiwilligen in der Vor- und Nachbereitung und auch während des Dienstes im Ausland begleitet werden können. Die RückkehrerInnen haben zudem die Möglichkeit, sich nach ihrer Rückkehr im

1 Die Tagung fand am 22. November 2013 in Stuttgart statt.

2 Der Dachverband Entwicklungspolitik Baden-Württemberg (DEAB) bildet sich aus 150 Eine-Welt-Initiativen, Weltläden, entwicklungspolitische Organisationen und Netzwerke in Baden-Württemberg bilden insgesamt ca. 300 Gruppen und Organisationen. Zentrale Aufgaben des 1975 gegründeten Dachverbands sind die Förderung seiner Mitglieder durch Information, Vernetzung, Fortbildung und die politische Vertretung ihrer Anliegen gegenüber Politik und Öffentlichkeit

3 finep ist eine gemeinnützige Projekt- und Beratungsorganisation im Bereich der Nachhaltigen Entwicklung mit Fokus auf den Themenfeldern Entwicklungspolitik, Umweltpolitik und Förderung der lokalen Demokratie. finep engagiert sich dabei gemeinsam mit Partnern in Deutschland und Europa seit 2010 im Bereich der RückkehrerInnenarbeit. 
Eine-Welt-Jugendnetzwerk OpenGlobe zu engagieren. Julia Brockmeier von CulturCooperation brachte Erfahrungen zur Verknüpfung von Freiwilligendiensten und Globalem Lernen aus dem internationalen Kontext ein: Durch Youth Camps mit europäischen Freiwilligen und Stakeholderkonferenzen mit Entsendern, Aufnahmeorganisationen aus dem globalen Süden, NROs der entwicklungspolitischen Bildungsarbeit und Freiwilligen findet ein gegenseitiger Austausch statt, um Engagement und Empowerment von Freiwilligen zu fördern. Auffällig sind die großen Unterschiede zwischen Freiwilligendiensten innerhalb Europas hinsichtlich Förderstrukturen, TeilnehmerInnenprofilen, Zielsetzungen, sowie der Wertschätzung von Freiwilligendiensten und von ehrenamtlichem Engagement in der Gesellschaft. finep hat die bundesweite, dienst- und trägerübergreifende Fortbildungsreihe „Zurück für die Zukunft“ für RückkehrerInnen entwickelt, die Interesse am Eine-Welt-Engagement haben. Kai Diederich präsentierte das Konzept, das neben Grundlagen zu entwicklungspolitischen Themen und Methoden des Globalen Lernens, Projektmanagement und Arbeitstechniken auch die Entwicklung und Umsetzung einer eigenen Projektidee durch die RückkehrerInnen umfasst. Ebenso wurden hilfreiche Arbeitsmaterialien aus dem EU-Projekt präsentiert wie zum Beispiel eine Interviewbroschüre und Handbücher für RückkehrerInnen.

Im Anschluss an die Präsentationen wurden verschiedene Fragestellungen des Vormittags im Rahmen von drei Workshops mit ReferentInnen und TeilnehmerInnen vertieft. Für die Vorbereitung von Freiwilligen auf ihren Auslandseinsatz wurde als Ergebnis festgehalten, dass es bei der pädagogischen Arbeit in dieser Phase insbesondere um die Bewusstseinsbildung über persönliche Entwicklung im Sinne der Selbsterkenntnis sowie der Schaffung von globalen Perspektiven geht. Globales Lernen in der Vorbereitung bedeutet: „Wie motiviere ich mein Herz, schaffe Perspektiven/Alternativen und entwickle das Handwerkszeug, das wichtig ist für den anstehenden Freiwilligendienst?" - und kann dabei die Lernprozesse der Freiwilligen unterstützen. Ehemalige Freiwillige spielen als TeamerInnen in der Vorbereitung eine wichtige Rolle, ausschlaggebend ist jedoch die Qualitätssicherung in deren Ausbildung.

In einem weiteren Workshop fand ein Austausch dazu statt, wie Engagement in der Nachbereitung von Freiwilligendiensten begünstigt werden kann. Den TeilnehmerInnen zufolge ist es besonders ratsam, die Erfahrungen aus dem Freiwilligendienst zu reflektieren, Interessengruppen zu bilden, freie Think Tanks aber auch konkrete, begrenzte Angebote zu bieten, sowie RückkehrerInnengruppen für andere (junge) Engagierte zu öffnen. Es wurde festgestellt, dass sich die Strukturen von Freiwilligendiensten ebenfalls auf das Engagement von RückkehrerInnen auswirken. Der großen Zahl an Freiwilligenplätzen und der vorwiegend einseitige Einsatz junger Deutscher im globalen Süden wohnt das Risiko inne, den Dienst als 
Abenteuerurlaub zu erleben und koloniale Strukturen zu reproduzieren. Für wichtig erscheinen daher eine kritische Diskussion mit den Freiwilligen über Rahmenbedingungen und eigene Motivation, der Austausch und ein gemeinsames Konzept zu Globalem und interkulturellem Lernen mit den Partnerorganisationen, sowie der Dialog mit Aufnahmeorganisationen und die Anerkennung deren Lernbegleitung. Auch RückkehrerInnen sollten als AkteurInnen in der Qualitätsverbesserung von Freiwilligendiensten stärker ernst genommen werden.

In einer dritten Gruppe wurde diskutiert, wie durch die Vernetzung von NROs der entwicklungspolitischen Inlandsarbeit und Entsendeorganisationen sowohl die Freiwilligen als auch die Organisationen selbst profitieren können. Ein Austausch von ReferentInnen kann die pädagogische Arbeit mit den Freiwilligen u. a. im Bereich des Globalen Lernens sowie der Vermittlung von Handwerkszeug für die entwicklungspolitische Inlandsarbeit stärken; Kontakte für weiteres Engagement der RückkehrerInnen eröffnen diesen neue Möglichkeiten. Die RückkehrerInnen wiederum können neue Zielgruppen für entwicklungspolitische Themen ansprechen und bringen authentische Erfahrungsberichte mit nach Deutschland. Hindernisse für die Zusammenarbeit sind zurzeit noch der geringe Dialog zwischen den AkteurInnen. Sinnvoll können daher Treffpunkte und Plattformen für die Vernetzung von RückkehrerInnen untereinander und der Kontakt zu Entsendeorganisationen und entwicklungspolitischen NROs sein. Bei einem Markt der Möglichkeiten in Form eines digitalen schwarzen Brettes könnten RückkehrerInnen und lokale Organisationen Kontakt zueinander aufnehmen - wichtig ist allerdings, dort Freiräume für junges Engagement zu schaffen. Kooperationsprojekte zwischen Entsendeorganisationen und NROs, z. B. mit einem Länder- oder Themenschwerpunkt, werden von den TeilnehmerInnen ebenfalls als erstrebenswert betrachtet. Bereits bei der Vorbereitung von Freiwilligen können hier Brücken für späteres Engagement geschlagen werden: Ein Kurzzeitpraktikum in der entwicklungspolitischen Inlandsarbeit eröffnet neue Perspektiven auf den anstehenden Freiwilligendienst und die Zeit danach.

Die Beiträge und Diskussionen der Tagung zeigen: RückkehrerInnen haben Lust auf Engagement in Deutschland, allerdings aus eigener Motivation, selbstbestimmt und zusammen mit Gleichgesinnten. Es wurde deutlich, dass unterschiedliche Engagementmöglichkeiten vorhanden sein sollten, sodass sich junge Menschen angepasst an ihre aktuelle Lebenssituation, ihr Zeitbudget und ihr Interesse einbringen können, z. B. auch punktuell und kurzfristig. Die Motivation steigt dabei durch die gemeinsamen Aktionen, die in der Praxis ein breites Spektrum aufweisen - von SMS-Aktionen, Kleidertauschpartys, Reste-Kochkursen bis hin zu Vorträgen, Schul-Workshops und Theateraufführungen. Unterstützt werden können die jungen Menschen dabei durch eine adäquate pädagogische Begleitung der individuellen Lernprozesse im Sinne des Globalen Lernens, beginnend bei der 
Vorbereitung des Freiwilligendienstes, sowie das Vermitteln von Handwerkszeug für Engagement (Grundlagen und Methoden des Globalen Lernens, Projektmanagement, Präsentationstechniken etc.) und die Unterstützung beim Fundraising. Eine Vernetzung von NROs der entwicklungspolitischen Inlandsarbeit, Entsendeorganisationen und (ehemaligen) Freiwilligen kann dabei für alle AkteurInnen fruchtbar sein und die Engagementmöglichkeiten der RückkehrerInnen erweitern.

Weitere Informationen erhalten Sie bei der Autorin dieses Beitrags sowie bei:

Kai Diederich, forum für internationale entwicklung + planung (finep)

kai.diederich@finep.org,www.finep.org 